\title{
Capsule Comment on Harrison et al., Assessing the Impact of Nurse Post-Discharge Telephone Calls on 30-day Hospital Readmission Rates
}

\author{
Jonathan C. Goldsmith, MD \\ Office of New Drugs, Center for Drug Evaluation and Research, Food and Drug Administration, Silver Spring, MD, USA.
}

$\mathrm{J}$ Gen Intern Med 29(11): 1533

DOI: $10.1007 / \mathrm{s} 11606-014-2972-0$

(c) Society of General Internal Medicine 2014

$\mathrm{T}$ he transition from an acute care hospital to a posthospitalization setting presents numerous logistical challenges. Failures in any of the aspects of the transition are associated with short-term (30-day) re-hospitalizations, emergency department (ED) visits, and increased costs of care. Currently, $20 \%$ of Medicare beneficiaries are readmitted within 30 days, ${ }^{1}$ and approximately $8 \%$ of all adults are readmitted within 30 days of an index hospital admission. ${ }^{2}$ Electronic medical records and electronic based ancillary services hold the promise of a more efficient and safer process to reduce readmissions and increase patient satisfaction.

Harrison and colleagues studied the use of a 72-hour telephone follow-up after acute care hospitalization to reduce hospital readmission rates. ${ }^{3}$ The study encountered several problems, including "no call attempted" to $25 \%$ of patients and call completion to less than $50 \%$ of discharged patients. The content of the post-discharge call is also not clear. In multivariable models, including a propensity score analysis, the authors identified a patient's ability to answer a telephone call as the strongest predictor of readmission and concurrently, the "success" of the program. This ability to answer the telephone is reminiscent of the relationship between lower hospital readmission rates and more favorable 6-minute walk test results in heart failure patients ${ }^{4}$ and global assessments of functional status performed in rehabilitation facilities. ${ }^{5}$ The ability to answer the phone emerged as a functional status proxy predicting both hospital readmission and the "success" of the program. The phone call itself had no effect.

Reducing avoidable readmissions is a laudable goal. Research suggests that avoidable readmissions can be reduced by improving discharge planning, care coordination, coaching, education and patient self-management. It is disappointing that the post-hospitalization telephone call had no effect in this study, as it theoretically could impact factors known to reduce readmissions. However, before abandoning post-discharge calls as ineffective, future research should focus on the goals these phone calls aim to achieve and the format and content of these calls. Further, the value and impact of the call intervention could be more rapidly and effectively evaluated by enriching the study population for patients with impaired functional status.

Conflicts of Interest: The author has no conflicts of interest with this article.

Corresponding Author: Jonathan C. Goldsmith, MD; Office of New Drugs, Center for Drug Evaluation and Research, Food and Drug Administration, Silver Spring, MD, USA (e-mail: Jonathan.goldsmith@fda.hhs.gov).

\section{REFERENCES}

1. Jencks SF, Williams MV, Coleman EA. Rehospitalizations among patients in the Medicare fee-for-service program. N Engl J Med. 2009;360(14):141828.

2. Sommers A, Cunningham PJ. Physician visits after hospital discharge: Implications for reducing readmissions. National Institute for Health Care Reform, Number 6, December 2011

3. Harrison JD, Auerbach A, Quinn K, Kynoch E, Mourad M. Assessing the impact of nurse post-discharge telephone calls on 30-day hospital readmission rates. J Gen Intern Med. (Article SPI \#2954).

4. Kommuri NV, Johnson ML, Koelling TM. Six-minute walk distance predicts 30-day readmission in hospitalized heart failure patients. Arch Med Res. 2010;41(5):363-8.

5. Hoyer EH, et al. Association of impaired functional status at hospital discharge and subsequent rehospitalization. J Hosp Med. 2014;9(5):277-82.

Published online September 3, 2014 